\title{
PREP-Mt: predictive RNA editor for plant mitochondrial genes Jeffrey P Mower*
}

\author{
Address: Department of Biology, Indiana University, Bloomington, IN, 47405, USA \\ Email: Jeffrey P Mower* - jpmower@indiana.edu \\ * Corresponding author
}

Published: 12 April 2005

BMC Bioinformatics 2005, 6:96 doi:10.1186/147/-2105-6-96
Received: 10 February 2005

Accepted: 12 April 2005

This article is available from: http://www.biomedcentral.com/l47I-2105/6/96

(c) 2005 Mower; licensee BioMed Central Ltd.

This is an Open Access article distributed under the terms of the Creative Commons Attribution License (http://creativecommons.org/licenses/by/2.0), which permits unrestricted use, distribution, and reproduction in any medium, provided the original work is properly cited.

\begin{abstract}
Background: In plants, RNA editing is a process that converts specific cytidines to uridines and uridines to cytidines in transcripts from virtually all mitochondrial protein-coding genes. There are thousands of plant mitochondrial genes in the sequence databases, but sites of RNA editing have not been determined for most. Accurate methods of RNA editing site prediction will be important in filling in this information gap and could reduce or even eliminate the need for experimental determination of editing sites for many sequences. Because RNA editing tends to increase protein conservation across species by "correcting" codons that specify unconserved amino acids, this principle can be used to predict editing sites by identifying positions where an RNA editing event would increase the conservation of a protein to homologues from other plants. PREP-Mt takes this approach to predict editing sites for any protein-coding gene in plant mitochondria.

Results: To test the general applicability of the PREP-Mt methodology, RNA editing sites were predicted for 370 full-length or nearly full-length DNA sequences and then compared to the known sites of RNA editing for these sequences. Of 60,263 cytidines in this test set, PREP-Mt correctly classified 58,994 as either an edited or unedited site (accuracy $=97.9 \%$ ). PREP-Mt properly identified 3,038 of the 3,698 known sites of RNA editing (sensitivity $=82.2 \%$ ) and 55,956 of the 56,565 known unedited sites (specificity $=98.9 \%$ ). Accuracy and sensitivity increased to $98.7 \%$ and $94.7 \%$, respectively, after excluding the 489 silent editing sites (which have no effect on protein sequence or function) from the test set.

Conclusion: These results indicate that PREP-Mt is effective at identifying $C$ to $U$ RNA editing sites in plant mitochondrial protein-coding genes. Thus, PREP-Mt should be useful in predicting protein sequences for use in molecular, biochemical, and phylogenetic analyses. In addition, PREPMt could be used to determine functionality of a mitochondrial gene or to identify particular sequences with unusual editing properties. The PREP-Mt methodology should be applicable to any system where RNA editing increases protein conservation across species.
\end{abstract}

\section{Background}

RNA editing is a type of RNA processing (such as polyadenylation, intron splicing, and 5' end-capping) that inserts, deletes, or modifies nucleotides in an RNA transcript, thereby changing the information encoded by the genome. First discovered in trypanosome mitochondria [1], RNA editing has since been observed in a range of eukaryotes, including slime molds, amoeboid protozoans, plants, animals, and fungi, and also in viruses $[2,3]$. In plants, RNA editing converts cytidines to uridines and 
uridines to cytidines in mitochondrial and plastid, but not nuclear, transcripts. The frequency and type of conversion in each organelle is highly lineage-specific [4-7]. In angiosperms, for example, approximately 400 editing sites (all C to $\mathrm{U}$ ) have been found in the 30 to 40 mitochondrial protein-coding genes [8-10], but only about 30 $\mathrm{C}$ to $\mathrm{U}$ sites were seen across over 100 plastid genes [1113]. In contrast, both types of conversion are found with high abundance in mitochondrial and plastid transcripts of ferns and hornworts $[4-6,14,15]$.

In all plant lineages, RNA editing most often alters the amino acid sequence encoded by protein-coding genes, but it can occasionally generate new start codons, create or remove stop codons, or cause silent changes that do not affect the protein sequence. RNA editing has also been observed in tRNA genes, untranslated regions, and introns [2], although the frequency of editing in these regions appears to be much lower. One feature observed immediately upon the discovery of RNA editing in plants is that edited transcripts code for proteins that are more conserved across species than proteins predicted from genomic DNA [16-18]. In fact, this tendency for codon "correction" was one of the clues that led to the discovery of RNA editing, since proteins predicted from early plant mitochondrial DNA sequences contained biochemically distinct amino acids at positions that are otherwise conserved throughout eukaryotes [16-18]. These initial observations have been repeatedly confirmed in almost all subsequent studies of editing in plants, with the most notable exceptions occurring in pseudogenes [19-21], which presumably have no selective constraints on their editing sites.

Because of the changes induced by RNA editing, the protein sequences encoded by mature mitochondrial transcripts are often quite different from what is encoded by the genomic DNA. In order to correctly analyze plant mitochondrial sequences in phylogenetic, molecular, or biochemical studies, RNA editing information must be known. Experimental determination, via direct comparison of the RNA transcript sequence and genomic DNA sequence, is the de facto standard for identification of sites of RNA editing. Given that these experimental analyses take time and cost money, however, two general approaches have been used to predict sites of RNA editing. The first relies on the possibility that the sequence context of an edited site contains information that signals the editing machinery or associated specificity factors. Indeed, experimental analyses of the surrounding sequence context indicate that nucleotides upstream and downstream are important in specifying sites of editing $[22,23]$. Furthermore, over $90 \%$ of editing sites have a pyrimidine at the adjacent upstream nucleotide $[8,24]$. Unfortunately, attempts at identifying consensus motifs beyond this one important nucleotide have met with little success $[8,24,25]$. The second predictive approach exploits the tendency of RNA editing to increase protein conservation across diverse taxa. Because of this "correcting" nature of RNA editing, it is possible to scan a protein sequence alignment for unconserved amino acids. Very often, when these unconserved amino acids have the potential to be corrected by RNA editing, they are actually edited. This approach has been shown to be very successful in predicting sites of RNA editing for several genes $[6,25,26]$, and has also been used to infer the absence of RNA editing in the entire mitochondrial genomes of Marchantia polymorpha, a complex thalloid liverwort, and the green algae Chara vulgaris and Chaetosphaeridium globosum [27-29]. Limited experimental evidence has so far corroborated the lack of editing in these lineages $[4-6,30]$.

In order to test the generality of the second predictive approach for any plant mitochondrial protein-coding gene, the PREP-Mt program was designed to predict editing sites using protein sequence comparisons and the correcting nature of RNA editing. Because the test results indicate that PREP-Mt is both fast and accurate, an online tool was also developed [31]. This resource should be useful now since editing sites have been experimentally determined for only a small percentage of plant mitochondrial genes available in the sequence databases, and it will become increasingly useful as more mitochondrial genomes get sequenced in the near future. PREP-Mt may also be effective in discriminating between functional genes and pseudogenes, as well as in elucidating mechanisms of RNA editing by exposing examples of genes that do not conform to the normal editing patterns in plant mitochondria.

\section{Implementation \\ Construction of the Aligned Sequence Database}

395 full-length or nearly full-length plant mitochondrial protein-coding genes, for which RNA editing sites have been experimentally determined or from organisms (Marchantia, Chara, and Chaetosphaeridium) inferred to lack RNA editing capability, were collected from Genbank. Gene sequences were extracted from each file and then edited according to Genbank annotations or literature sources. The edited gene sequences were translated into proteins according to the standard genetic code. Homologous proteins were aligned using ClustalW version 1.81 [32] and manually adjusted when necessary. The Aligned Sequence Database (ASD) consisted of these protein sequence alignments (see additional files 1, 2, 3, $4,5,6,7,8,9,10,11,12,13,14,15,16,17,18,19,20$, $21,22,23,24,25,26,27,28,29,30,31,32,33,34,35$, $36,37,38,39,40,41,42$ for alignments). 
In some cases, editing site annotations in the Genbank files were associated with nucleotides that were not a cytidine. These incorrect annotations were usually the result of obvious human errors and were corrected by referring to the literature sources prior to inclusion in the database. In addition, nine sequences from Marchantia (atp4, atp8, $c c m F c-A, c c m F c-B, c c m F n, r p l 2, r p s 1, r p s 3, r p s 4)$, eight from Chara (atp4, atp8, ccmF, rpl2, rpl5, rps1, rps3, rps4), and seven from Chaetosphaeridium (atp4, atp8, rpl2, rpl5, rps1, $r p s 3, r p s 4$ ) aligned poorly to the other ASD sequences. Because the PREP-Mt program relies on accurate alignments to determine RNA editing sites, these divergent proteins were not included. For the same reason, nonhomologous $5^{\prime}$ and $3^{\prime}$ extensions present in some mitochondrial proteins (e.g., atp6 and $r p s 2$ ) were trimmed from the alignments. After removal of the divergent sequences, 371 sequences remained in the final alignments, spanning all 42 known protein-coding genes variably present in land plant mitochondria [33]. There were 8.8 sequences in each alignment on average, with the actual numbers ranging from 22 sequences for nad 3 to only a single Marchantia sequence for $r p s 8$.

\section{Algorithm for RNA editing site prediction}

Given a protein-coding DNA sequence and its gene identity, PREP-Mt predicts sites of C to U RNA editing [31]. The input sequence is translated using the standard genetic code and then aligned to the homologous ASD alignment with ClustalW using default parameters and the quicktree option. Next, for each column in the protein alignment, the corresponding codon from the input DNA sequence is examined to determine whether editing is possible. If the codon contains one or more cytidines, then the set of all possible unedited and edited states for that codon is determined. For example, if the input DNA sequence contained the codon "CCG", then the set of possible states in the RNA transcript would be "CCG" (not edited), "UCG" (edited at first position), "CUG" (edited at second position), and "UUG" (edited at first and second position). The amino acid, $i$, encoded by each of the possible codon states is compared to the amino acid, $j$, from all $N$ database sequences. The score for each state, $S_{i^{\prime}}$ is then defined by the equation

$$
S_{i}=\frac{1}{N} \sum_{\text {all } j} M_{i j}
$$

where the match parameter, $M_{i j}$, is determined by

$$
M_{i j}= \begin{cases}1 & i \text { and } j \text { are the same } \\ 0 & i \text { and } j \text { are different }\end{cases}
$$

Thus, the score for each possible state is a value that ranges from 0 to 1 and is simply the percentage of matches to the amino acids in the ASD sequences for that column. The

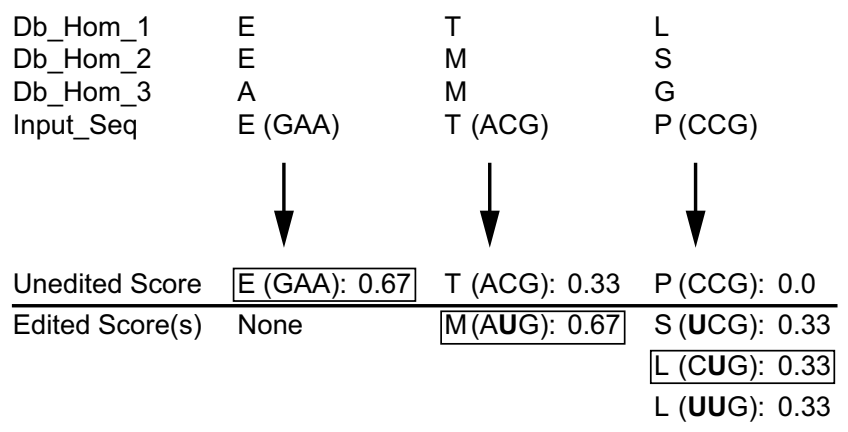

\section{Figure I}

Score calculation and prediction of RNA editing. The hypothetical alignment shows an input sequence aligned to three homologues from the Aligned Sequence Database. The predicted state is boxed. Column I shows a case where RNA editing is not possible. Column 2 presents a case where the editing state is more strongly supported. Column 3 demonstrates the various rules placed on the algorithm. The CUG is taken as the correct state because it requires fewer edits than UUG and is edited at the second position, whereas UCG is edited at the first position.

state with the highest score is reported as the predicted state. In case of a tie, the state that requires the fewest number of edits is chosen as the predicted state, since the vast majority of cytidines in plant mitochondrial genes are not actually edited. Based on this rule, silent editing sites are always disfavored because, by definition, they do not affect the encoded amino acid and would therefore always tie a state that had fewer editing sites. If a tie occurs between states that require an equal number of edits, the state that is edited at the second codon position is chosen as the predicted state, since approximately $50 \%$ of all editing sites in plants occur at the second position $[8,24]$. An example of the scoring scheme is presented in Figure 1.

As an additional requirement, a cutoff value, $C$, can be enforced. If a cutoff value is specified, $S_{i}$ for an edited state must be greater than or equal to $C$ in order to be reported as the predicted state. Thus, PREP-Mt would predict an unedited state for a particular codon if $S_{i}$ for the edited state is less than $C$, even if the edited state has a higher $S_{i}$ than the unedited state. $C$ must be a value ranging from 0 to 1 .

\section{PREP-Mt performance analyses}

To evaluate the predictive performance of PREP-Mt, each sequence in the ASD was used as a test case. First, the protein sequence of the test case was removed from the ASD so that it would not be tested against itself. Then, the fulllength protein-coding region for the test case was 
collected from its Genbank file and this unedited DNA sequence was used as input into PREP-Mt. Sites of RNA editing predicted by PREP-Mt were scored as either correct (TP, true positive) or incorrect ( $F P$, false positive) based on comparison to the known edited sites. Similarly, sites predicted to remain unedited were scored as either correct ( $T N$, true negative) or incorrect ( $F N$, false negative) after comparison to the known unedited sites. This process was repeated for each sequence in the ASD, except for the single $r p s 8$ sequence which could not be tested because there were no other $r p s 8$ sequences to test against. Using the above classification, several statistical measures of predictive performance were calculated:

$$
\begin{aligned}
& \text { Sensitivity }=\frac{T P}{T P+F N} \\
& \text { Specificity }=\frac{T N}{T N+F P} \\
& \text { Accuracy }=\frac{T P+T N}{T P+T N+F P+F N}
\end{aligned}
$$

Because the number of known edited sites was proportionately much lower than the number of known unedited sites, the accuracy value was highly dependent on the specificity value. To determine the expected accuracy if the number of known edited and unedited sites were in equal proportion, a balanced accuracy statistic was also calculated according to the formula

$$
\text { Balanced Accuracy }=\frac{\text { Sensitivity }+ \text { Specificity }}{2}
$$

Finally, to evaluate the effect of the cutoff value on the predictive performance of PREP-Mt, the performance analysis described above was rerun with $C$ values ranging from 0.1 to 1.0 at all increments of 0.1 .

\section{Results \\ Classification of known editing sites}

There were 60,263 cytidines present in the 370 tested protein-coding sequences (the single rps 8 gene could not be tested because there were no $r p s 8$ homologues to test against). For sequences from the complete mitochondrial genomes of Marchantia, Chara, and Chaetosphaeridium, RNA editing capability was assumed to be absent, as indicated by predictive and experimental analyses [4-6,27$30]$. For the remaining sequences, sites of RNA editing had been determined experimentally and were reported in the Genbank files and/or the literature. Based on these sources, 3,698 (6.1\%) of the cytidines were classified as known sites of RNA editing, while the remaining 56,565 (93.9\%) were classified as known unedited cytidines.

\section{PREP-Mt performance analyses}

PREP-Mt was used to predict editing sites for all 370 sequences in the test set. PREP-Mt's predictive performance was measured by comparing the predicted state of each cytidine to its known state (Tables 1 and 2). Of the 3,698 known edited sites, PREP-Mt correctly identified 3,038 (TP) as edited sites and incorrectly predicted 660 $(F N)$ as unedited sites (sensitivity $=82.2 \%)$. PREP-Mt also correctly identified 55,956 (TN) out of the 56,565 known unedited sites, while incorrectly predicting 609 (FP) to be edited (specificity $=98.9 \%$ ). Altogether, PREP-Mt correctly classified 58,994 of the 60,263 cytidines in the test sequences as either an edited or unedited position (accuracy $=97.9 \%$, balanced accuracy $=90.5 \%)$. Of the 660 false negatives, 489 occurred at first or third codon positions that did not change the amino acid encoded by the codon. Excluding these silent editing positions, which have no effect on protein sequence or function, sensitivity increased to $94.7 \%$, accuracy increased to $98.7 \%$, and balanced accuracy increased to $96.8 \%$. Specificity was unaffected by the silent site adjustment. The speed of prediction was also very fast. For each of the 370 tested sequences, editing site prediction took less than one second on a $3.2 \mathrm{GHz}$ Pentium IV computer running RedHat Linux 9 with 1 GB of RAM (data not shown).

To evaluate the predictive performance of PREP-Mt in more detail, the performance results were subdivided by gene (Table 1) and by genus (Table 2). After doing so, it was apparent that specificity remained very high for all treatments, never falling below 95\% for any gene or genus. Accuracy was also consistently high, with only a single case lower than 90\%. In contrast, sensitivity was dependent on the gene or genus analyzed. In particular, several genes (sdh3, sdh4, atp8, rpl2, rps1, rps3, and rps19) and genera (Gymnocladus, Nicotiana, Oxalis, Podophyllum, and Secale) exhibited low sensitivity scores. In some cases, the poor sensitivity scores were due to the fact that a large portion of the known edited sites were at silent positions and therefore could not be predicted by PREP-Mt. Calculating sensitivity after excluding the silent edited sites helped to alleviate many of these low scores, and in general greatly increased sensitivity scores for most genes and genera. In addition, low sensitivity may be the result of the small sample sizes found for most of the poorly performing examples, if by chance the small set of genes or genera sampled does not conform to the normal editing patterns of plant mitochondrial genes. In this regard, it is interesting to note that the editing data for Gymnocladus, Oxalis, and Podophyllum came from sdh3 and sdh4. It is possible that the low sensitivities seen for Gymnocladus, Oxalis, and Podophyllum were the result of sampling from these poorly performing genes, or, conversely, that the low scores for $s d h 3$ and $s d h 4$ were due to the inclusion of these poorly performing genera. 
Table I: PREP-Mt performance results subdivided by gene

\begin{tabular}{|c|c|c|c|c|c|c|c|c|c|}
\hline Gene & $\begin{array}{l}\text { No. of } \\
\text { Species }\end{array}$ & $\mathrm{TP}$ & $\mathrm{TN}$ & FP & FN & Specificity & Sensitivity & Accuracy & $\begin{array}{l}\text { Balanced } \\
\text { Accuracy }\end{array}$ \\
\hline \multicolumn{10}{|c|}{ Complex I } \\
\hline nadl & 10 & 135 & 1632 & 14 & $33(2)$ & 99.1 & $80.4(98.5)$ & $97.4(99.1)$ & $89.8(98.8)$ \\
\hline nad2 & 9 & 140 & 2337 & 22 & $42(9)$ & 99.1 & $76.9(94.0)$ & $97.5(98.8)$ & $88.0(96.5)$ \\
\hline nad3 & 22 & 274 & 1217 & 8 & $47(4)$ & 99.3 & $85.4(98.6)$ & $96.4(99.2)$ & 92.4 (99.0) \\
\hline nad4 & 9 & 133 & 2358 & 19 & $12(I)$ & 99.2 & 91.7 (99.3) & $98.8(99.2)$ & 95.5 (99.2) \\
\hline nad4L & 8 & 49 & 303 & 5 & $0(0)$ & 98.4 & $100.0(100.0)$ & $98.6(98.6)$ & 99.2 (99.2) \\
\hline nad5 & 10 & 122 & 3647 & 29 & $10(4)$ & 99.2 & $92.4(96.8)$ & $99.0(99.1)$ & 95.8 (98.0) \\
\hline nad6 & 10 & 70 & 995 & 16 & $15(3)$ & 98.4 & 82.4 (95.9) & $97.2(98.2)$ & 90.4 (97.2) \\
\hline nad7 & 8 & 117 & 1655 & 5 & $22(I)$ & 99.7 & $84.2(99.2)$ & $98.5(99.7)$ & 91.9 (99.4) \\
\hline nad9 & 12 & 75 & 1236 & 7 & $5(3)$ & 99.4 & $93.8(96.2)$ & 99.1 (99.2) & $96.6(97.8)$ \\
\hline \multicolumn{10}{|c|}{ Complex II } \\
\hline sdh3 & 7 & 6 & 391 & 12 & II (2) & 97.0 & $35.3(75.0)$ & $94.5(96.6)$ & $66.2(86.0)$ \\
\hline sdh4 & 8 & 10 & 361 & 14 & $16(3)$ & 96.3 & 38.5 (76.9) & $92.5(95.6)$ & $67.4(86.6)$ \\
\hline \multicolumn{10}{|c|}{ Complex III } \\
\hline$c o b$ & 13 & 117 & 2834 & 19 & $25(8)$ & 99.3 & $82.4(93.6)$ & 98.5 (99.1) & 90.9 (96.5) \\
\hline \multicolumn{10}{|c|}{ Complex IV } \\
\hline $\operatorname{cox} I$ & 9 & 38 & 2936 & 7 & $7(5)$ & 99.8 & $84.4(88.4)$ & $99.5(99.6)$ & 92.1 (94.I) \\
\hline $\operatorname{cox} 2$ & 19 & 193 & 2644 & 22 & $33(\mathrm{II})$ & 99.2 & $85.4(94.6)$ & 98.1 (98.9) & $92.3(96.9)$ \\
\hline $\operatorname{cox} 3$ & 12 & 80 & 1793 & 21 & II (2) & 98.8 & $87.9(97.6)$ & $98.3(98.8)$ & 93.4 (98.2) \\
\hline \multicolumn{10}{|c|}{ Complex V } \\
\hline atpl & 10 & 21 & 2926 & 10 & $8(I)$ & 99.7 & $72.4(95.5)$ & $99.4(99.6)$ & $86.0(97.6)$ \\
\hline atp 4 & 7 & 51 & 730 & 21 & $18(8)$ & 97.2 & $73.9(86.4)$ & $95.2(96.4)$ & $85.6(91.8)$ \\
\hline atp6 & 12 & 88 & 1893 & II & $10(2)$ & 99.4 & $89.8(97.8)$ & $99.0(99.3)$ & $94.6(98.6)$ \\
\hline atp8 & 8 & 16 & 757 & 10 & $10(2)$ & 98.7 & $61.5(88.9)$ & 97.5 (98.5) & 80.1 (93.8) \\
\hline atp9 & 19 & 91 & 792 & 1 & $17(0)$ & 99.9 & $84.3(100.0)$ & $98.0(99.9)$ & 92.1 (99.9) \\
\hline \multicolumn{10}{|c|}{ Cytochrome $\mathrm{c}$ biogenesis } \\
\hline $\mathrm{ccmB}$ & 9 & 216 & 1013 & 14 & $63(6)$ & 98.6 & $77.4(97.3)$ & 94.1 (98.4) & $88.0(98.0)$ \\
\hline $\mathrm{ccmC}$ & 9 & 187 & 1212 & 24 & $26(I)$ & 98.1 & $87.8(99.5)$ & $96.5(98.2)$ & $92.9(98.8)$ \\
\hline $\mathrm{ccmFc}$ & 6 & 84 & 1631 & 23 & $30(10)$ & 98.6 & $73.7(89.4)$ & $97.0(98.1)$ & $86.1(94.0)$ \\
\hline $\mathrm{ccmFn}$ & 6 & 155 & 2249 & 39 & $38(2 I)$ & 98.3 & $80.3(88.1)$ & $96.9(97.6)$ & $89.3(93.2)$ \\
\hline \multicolumn{10}{|c|}{ Ribosomal proteins } \\
\hline$r p / 2$ & 4 & 2 & 1057 & 14 & $6(4)$ & 98.7 & $25.0(33.3)$ & 98.1 (98.3) & $61.8(66.0)$ \\
\hline$r p / 5$ & 9 & 45 & 919 & 14 & $5(3)$ & 98.5 & 90.0 & 98.1 (98.3) & $94.2(96.1)$ \\
\hline rpl6 & 3 & 0 & 138 & 3 & $0(0)$ & 97.9 & - & $97.9(97.9)$ & - \\
\hline rpll6 & 9 & 33 & 692 & 6 & $13(2)$ & 99.1 & $71.7(94.3)$ & $97.4(98.9)$ & 85.4 (96.7) \\
\hline rpsl & 3 & 2 & 295 & 4 & $5(5)$ & 98.7 & $28.6(28.6)$ & 97.1 (97.1) & $63.6(63.6)$ \\
\hline rps2 & 7 & 25 & 1489 & 15 & $7(5)$ & 99.0 & $78.1(83.3)$ & 98.6 (98.7) & 88.6 (91.2) \\
\hline rps3 & 7 & 54 & 2183 & 19 & $33(\mathrm{II})$ & 99.1 & $62.1(83.1)$ & 97.7 (98.7) & 80.6 (91.I) \\
\hline rps4 & 6 & 83 & 1116 & 22 & $18(6)$ & 98.1 & $82.2(93.3)$ & $96.8(97.7)$ & 90.1 (95.7) \\
\hline rps7 & 8 & 6 & 664 & 19 & $2(1)$ & 97.2 & $75.0(85.7)$ & $97.0(97.1)$ & 86.1 (91.5) \\
\hline rps 10 & 5 & 8 & 266 & 9 & $0(0)$ & 96.7 & $100.0(100.0)$ & $96.8(96.8)$ & 98.4 (98.4) \\
\hline rps II & 3 & 0 & 222 & I & $0(0)$ & 99.6 & - & $99.6(99.6)$ & - \\
\hline $\mathrm{rps} / 2$ & 17 & 87 & 1142 & 13 & $5(1)$ & 98.9 & 94.6 (98.9) & $98.6(98.9)$ & 96.7 (98.9) \\
\hline rps $/ 3$ & 10 & 34 & 554 & 8 & $5(2)$ & 98.6 & $87.2(94.4)$ & $97.8(98.3)$ & $92.9(96.5)$ \\
\hline rps $/ 4$ & 5 & 2 & 239 & 7 & $0(0)$ & 97.2 & $100.0(100.0)$ & $(97.2)(97.2)$ & $98.6(98.6)$ \\
\hline rps 19 & 6 & 16 & 242 & 11 & $9(3)$ & 95.7 & $64.0(84.2)$ & $92.8(94.9)$ & 79.8 (89.9) \\
\hline \multicolumn{10}{|l|}{ Other } \\
\hline matR & 8 & 73 & 4109 & 30 & $20(7)$ & 99.3 & 78.5 (9I.3) & $98.8(99.1)$ & 88.9 (95.3) \\
\hline$m t t B$ & 8 & 100 & 1087 & 41 & $23(12)$ & 96.4 & $81.3(89.3)$ & 94.9 (95.7) & $88.8(92.8)$ \\
\hline Overall & 370 & 3,038 & 55,956 & 609 & $660(171)$ & 98.9 & $82.2(94.7)$ & 97.9 (98.7) & $90.5(96.8)$ \\
\hline
\end{tabular}

Values after exclusion of silent editing sites are shown in parentheses. In some cases (marked with a '-'), sensitivity and balanced accuracy could not be calculated because there were no known edited sites (i.e., $\mathrm{TP}+\mathrm{FN}=0$ ). 
Table 2: PREP-Mt performance results subdivided by genus

\begin{tabular}{|c|c|c|c|c|c|c|c|c|c|}
\hline Genus & No. of Genes & $\mathrm{TP}$ & $\mathrm{TN}$ & FP & $\mathrm{FN}$ & Specificity & Sensitivity & Accuracy & $\begin{array}{l}\text { Balanced } \\
\text { Accuracy }\end{array}$ \\
\hline \multicolumn{10}{|l|}{ Angiosperms } \\
\hline \multicolumn{10}{|l|}{ Eudicots } \\
\hline Amphicarpaea & 1 & 13 & 143 & 0 & $0(0)$ & 100.0 & $100.0(100.0)$ & $100.0(100.0)$ & $100.0(100.0)$ \\
\hline Arabidopsis & 31 & 344 & 5880 & 58 & $89(24)$ & 99.0 & 79.4 (93.5) & 97.7 (98.7) & $89.2(96.3)$ \\
\hline Beta & 30 & 299 & 5406 & 52 & $50(\mathrm{II})$ & 99.0 & $85.7(96.5)$ & $98.2(98.9)$ & $92.4(97.7)$ \\
\hline Brassica & 32 & 362 & 5874 & 53 & $55(15)$ & 99.1 & $86.8(96.0)$ & $98.3(98.9)$ & $93.0(97.6)$ \\
\hline Cologania & 1 & 13 & 138 & 1 & I (0) & 99.3 & $92.9(100.0)$ & $98.7(99.3)$ & 96.1 (99.6) \\
\hline Cucumis & 2 & 19 & 332 & 7 & $0(0)$ & 97.9 & $100.0(100.0)$ & $98.0(98.0)$ & $99.0(99.0)$ \\
\hline Daucus & 5 & 42 & 820 & 4 & $9(4)$ & 99.5 & 82.4 (9I.3) & $98.5(99.1)$ & $90.9(95.4)$ \\
\hline Dumasia & 1 & 14 & $|4|$ & 0 & I (0) & 100.0 & $93.3(100.0)$ & $99.4(100.0)$ & $96.7(100.0)$ \\
\hline Euphorbia & 1 & 1 & 53 & 0 & $0(0)$ & 100.0 & $100.0(100.0)$ & $100.0(100.0)$ & $100.0(100.0)$ \\
\hline Glycine & 2 & 14 & 566 & 2 & $2(0)$ & 99.6 & $87.5(100.0)$ & 99.3 (99.7) & $93.6(99.8)$ \\
\hline Gymnocladus & 2 & 1 & 108 & 4 & $2(1)$ & 96.4 & $33.3(50.0)$ & $94.8(95.6)$ & $64.9(73.2)$ \\
\hline Helianthus & 4 & 39 & 362 & I & $3(1)$ & 99.7 & 92.9 (97.5) & 99.0 (99.5) & $96.3(98.6)$ \\
\hline Lactuca & 1 & 16 & 282 & I & $0(0)$ & 99.6 & $100.0(100.0)$ & 99.7 (99.7) & $99.8(99.8)$ \\
\hline Lespedeza & 1 & 13 & 142 & 0 & $0(0)$ & 100.0 & $100.0(100.0)$ & $100.0(100.0)$ & $100.0(100.0)$ \\
\hline Lupinus & 6 & 68 & 484 & 3 & $3(0)$ & 99.4 & $95.8(100.0)$ & $98.9(99.5)$ & $97.6(99.7)$ \\
\hline Lycopersicon & 4 & 52 & 550 & I & $21(3)$ & 99.8 & $71.2(94.5)$ & $96.5(99.3)$ & 85.5 (97.2) \\
\hline Malus & 1 & 8 & 47 & 0 & $0(0)$ & 100.0 & $100.0(100.0)$ & $100.0(100.0)$ & $100.0(100.0)$ \\
\hline Nicotiana & 2 & 17 & 258 & 1 & $12(3)$ & 99.6 & $58.6(85.0)$ & $95.5(98.6)$ & $79.1(92.3)$ \\
\hline Oenothera & 23 & 283 & 4395 & 58 & $95(34)$ & 98.7 & $74.9(89.3)$ & $96.8(98.1)$ & $86.8(94.0)$ \\
\hline Olea & 2 & 11 & 249 & 0 & I (0) & 100.0 & 91.7 (100.0) & $99.6(100.0)$ & $95.8(100.0)$ \\
\hline Oxalis & 1 & 2 & 51 & 0 & $3(1)$ & 100.0 & $40.0(66.7)$ & $94.6(98.1)$ & $70.0(83.3)$ \\
\hline Petunia & 9 & 84 & 1132 & 8 & $24(5)$ & 99.3 & $77.8(94.4)$ & 97.4 (98.9) & $88.5(96.8)$ \\
\hline Pisum & 4 & 53 & 487 & 4 & $8(0)$ & 99.2 & $86.9(100.0)$ & $97.8(99.3)$ & $93.0(99.6)$ \\
\hline Podophyllum & 2 & 3 & 96 & 5 & $8(0)$ & 95.0 & $27.3(100.0)$ & 88.4 (95.2) & $61.2(97.5)$ \\
\hline Raphanus & 5 & 31 & 453 & 3 & $6(1)$ & 99.3 & $83.8(96.9)$ & $98.2(99.2)$ & $91.6(98.1)$ \\
\hline Solanum & 8 & 58 & 1259 & 16 & $21(9)$ & 98.7 & $73.4(86.6)$ & $97.3(98.1)$ & 86.1 (92.7) \\
\hline Vitis & 1 & 20 & 224 & 1 & I (0) & 99.6 & $95.2(100.0)$ & $99.2(99.6)$ & $97.4(99.8)$ \\
\hline \multicolumn{10}{|l|}{ Magnoliids } \\
\hline Magnolia & 5 & 79 & 527 & 2 & $12(3)$ & 99.6 & $86.8(96.3)$ & 97.7 (99.2) & $93.2(98.0)$ \\
\hline \multicolumn{10}{|l|}{ Monocots } \\
\hline Acorus & 1 & 8 & 143 & 2 & $0(0)$ & 98.6 & $100.0(100.0)$ & 98.7 (98.7) & $99.3(99.3)$ \\
\hline Allium & 2 & 24 & $|3|$ & 2 & $0(0)$ & 98.5 & $100.0(100.0)$ & 98.7 (98.7) & $99.2(99.2)$ \\
\hline Coix & 2 & 23 & 121 & 0 & $3(0)$ & 100.0 & $88.5(100.0)$ & $98.0(100.0)$ & $94.2(100.0)$ \\
\hline Elymus & 1 & 12 & 157 & 2 & $0(0)$ & 98.7 & $100.0(100.0)$ & $98.8(98.8)$ & $99.4(99.4)$ \\
\hline Hordeum & 2 & 12 & 388 & 1 & $3(1)$ & 99.7 & $80.0(92.3)$ & $99.0(99.5)$ & $89.9(96.0)$ \\
\hline Oryza & 34 & 395 & 5919 & 71 & $90(28)$ & 98.8 & $81.4(93.4)$ & $97.5(98.5)$ & 90.1 (96.1) \\
\hline Ruscus & 1 & 16 & 146 & 1 & $3(2)$ & 99.3 & $84.2(88.9)$ & $97.6(98.2)$ & $91.8(94.1)$ \\
\hline Secale & 2 & 6 & 353 & 5 & $3(0)$ & 98.6 & $66.7(100.0)$ & $97.8(98.6)$ & $82.6(99.3)$ \\
\hline Sorghum & 5 & 52 & 479 & 4 & $12(1)$ & 99.2 & $81.3(98.1)$ & $97.1(99.1)$ & $90.2(98.6)$ \\
\hline Triticum & 26 & 360 & 4748 & 36 & $79(13)$ & 99.2 & $82.0(96.5)$ & $97.8(99.0)$ & $90.6(97.9)$ \\
\hline Zea & 11 & 104 & 1933 & 16 & $23(7)$ & 99.2 & $81.9(93.7)$ & 98.1 (98.9) & $90.5(96.4)$ \\
\hline \multicolumn{10}{|l|}{ Gymnosperms } \\
\hline Cycas & 3 & 35 & 444 & 7 & $14(4)$ & 98.4 & $71.4(89.7)$ & $95.8(97.8)$ & $84.9(94.1)$ \\
\hline Pinus & 2 & 32 & 123 & 5 & $3(0)$ & 96.1 & $91.4(100.0)$ & 95.1 (96.9) & $93.8(98.0)$ \\
\hline \multicolumn{10}{|l|}{ Other } \\
\hline Chaetosphaeridium & 29 & 0 & 3011 & 49 & $0(0)$ & 98.4 & - & $98.4(98.4)$ & - \\
\hline Chara & 30 & 0 & 3549 & 72 & $0(0)$ & 98.0 & - & $98.0(98.0)$ & - \\
\hline Marchantia & 32 & 0 & 3852 & 51 & $0(0)$ & 98.7 & - & 98.7 (98.7) & - \\
\hline Overall & 370 & $\begin{array}{r}3,03 \\
8\end{array}$ & $\begin{array}{r}55,95 \\
6\end{array}$ & $\begin{array}{r}60 \\
9\end{array}$ & $\begin{array}{r}660 \\
(171)\end{array}$ & 98.9 & $82.2(94.7)$ & $97.9(98.7)$ & $90.5(96.8)$ \\
\hline
\end{tabular}

Notes are the same as for Table I. 
PREP-Mt predictive performance was also affected by the inclusion of sequences with unusually poor predictive results. 172 of the 609 false positives were found in genes from the three organisms (Marchantia, Chara, and Chaetosphaeridium) assumed to lack the ability to edit their transcripts (Table 2). The numbers of false positives across these three genomes were similar to those from the four complete genomes (Arabidopsis, Beta, Brassica, and Oryza) that do edit their transcripts, which is most likely reflective of an underlying rate of false positive prediction by PREPMt. However, if the assumption that Marchantia, Chara, and Chaetosphaeridium lack RNA editing is not correct, then some of these false positives sites may be true sites of editing. Further experimental analysis is needed to verify that RNA editing is absent in these three species. Another large portion of the false positives came from individual sequences that demonstrated poor predictive results (Table 3). Of the 58 predicted editing sites from 12 examples, 54 were false positives. On average, the false positives received moderately to very high predictive scores, making it unlikely that these examples are a collection of sequences that have a large number of spurious predictions by chance. These examples could simply be the result of incomplete experimental analysis, annotation errors, or pseudogenization. More interestingly, they may represent a small class of functional mitochondrial sequences that exhibit unusual editing properties for as yet undetermined reasons.

\section{Effect of the cutoff score on editing site prediction}

The predictive analyses presented above did not impose a cutoff value (i.e., $C=0$ ). To determine the effect of imposing a minimum cutoff value on editing site prediction, the predictive analyses were reevaluated using $C$ values ranging from 0.1 to 1.0 (Fig. 2). As expected, increasing the cutoff value led to a decrease in the number of false positives and an increase in the number of false negatives. Up to $C=0.6$, this was a balanced trade-off, because the number of false positives that decreased was approximately equal to the number of increased false negatives. As $C$ was increased from 0.6 to 1.0 , however, false negatives accumulated much faster than false positives diminished. Consequently, prediction accuracy remained roughly constant as the $C$ value was increased to 0.6 , but then sharply fell upon further increases of $C$. Because specificity is inversely related to the number of false positives (and is not affected by the number of false negatives), the specificity and false positive curves are also inversely related. Likewise, the sensitivity and false negative curves are inversely proportional to one another.

\section{Discussion PREP-Mt performance analysis}

The PREP-Mt program is very accurate, correctly classifying $98 \%$ of all cytidines as either an edited or unedited site
Table 3: Examples of unusually poor predictive results

\begin{tabular}{|c|c|c|c|c|c|}
\hline Gene & Species & TP & FP & $\mathrm{FN}$ & Score \\
\hline$a t p l$ & Secale cereale & 0 & 5 & $I(0)$ & 0.96 \\
\hline $\operatorname{cox} 3$ & Oryza sativa & I & 13 & $0(0)$ & 0.93 \\
\hline$r p / 2$ & Arabidopsis thaliana & 0 & 3 & I (0) & 0.78 \\
\hline$r p / 2$ & Brassica napus & 1 & 3 & $I(0)$ & 0.78 \\
\hline$r p / 2$ & Oenothera berteriana & I & 5 & $3(3)$ & 0.67 \\
\hline$r p / 2$ & Oryza sativa & 0 & 3 & $I(I)$ & 0.67 \\
\hline rpsl & Oenothera berteriana & 0 & 3 & $0(0)$ & 1.00 \\
\hline rps 7 & Arabidopsis thaliana & 0 & 2 & $0(0)$ & 0.93 \\
\hline $\operatorname{rps} / 2$ & Oryza sativa & 0 & 6 & $0(0)$ & 0.95 \\
\hline rps $/ 4$ & Brassica napus & 0 & 3 & $0(0)$ & 0.67 \\
\hline sdh4 & Gymnocladus dioica & 0 & 4 & $0(0)$ & 0.68 \\
\hline sdh4 & Podophyllum peltatum & 1 & 4 & $4(0)$ & 0.68 \\
\hline Overall & & 4 & 54 & II (4) & 0.83 \\
\hline
\end{tabular}

Predicted score is averaged across all incorrectly predicted edited sites (FP). The numbers of FN after exclusion of silent editing sites are shown in parentheses.

from 370 sequences spanning 41 functionally distinct genes and 44 diverse genera. PREP-Mt is also extremely specific and highly sensitive (at least for non-silent editing sites), properly identifying $99 \%$ of the known unedited sites and 95\% of the known edited sites that change the amino acid encoded by a codon. Because these results are consistent for almost all genes and genera, the PREP-Mt methodology appears to be generally applicable for plant mitochondrial protein-coding genes. Furthermore, predictions are made exceptionally fast, which makes this program appropriate as an online resource. The speed of PREP-Mt is primarily due to the facts that the ASD homologues are prealigned, the number of sequences in each alignment is low, and mitochondrial genes are less than 1,000 nucleotides in length on average.

The high specificity of PREP-Mt is not surprising, because the methodology takes advantage of the extraordinary conservation (with rare exception) of plant mitochondrial sequences, which have the lowest known substitution rates for any organism [[34,35]; but see ref. [36]], and the limited range of amino acids possibly produced after RNA editing of a specific codon, which is usually only one out of 20. Thus, it is very unlikely that a column in a protein alignment will lead to a spurious prediction by chance. However, in poorly conserved regions of a protein alignment, one or two sequences in the ASD could by chance have the specific amino acid encoded by the edited state of an input sequence codon. As an example, initial analyses of PREP-Mt performance did not trim the non-homologous 5' and 3' extensions found in several mitochondrial 

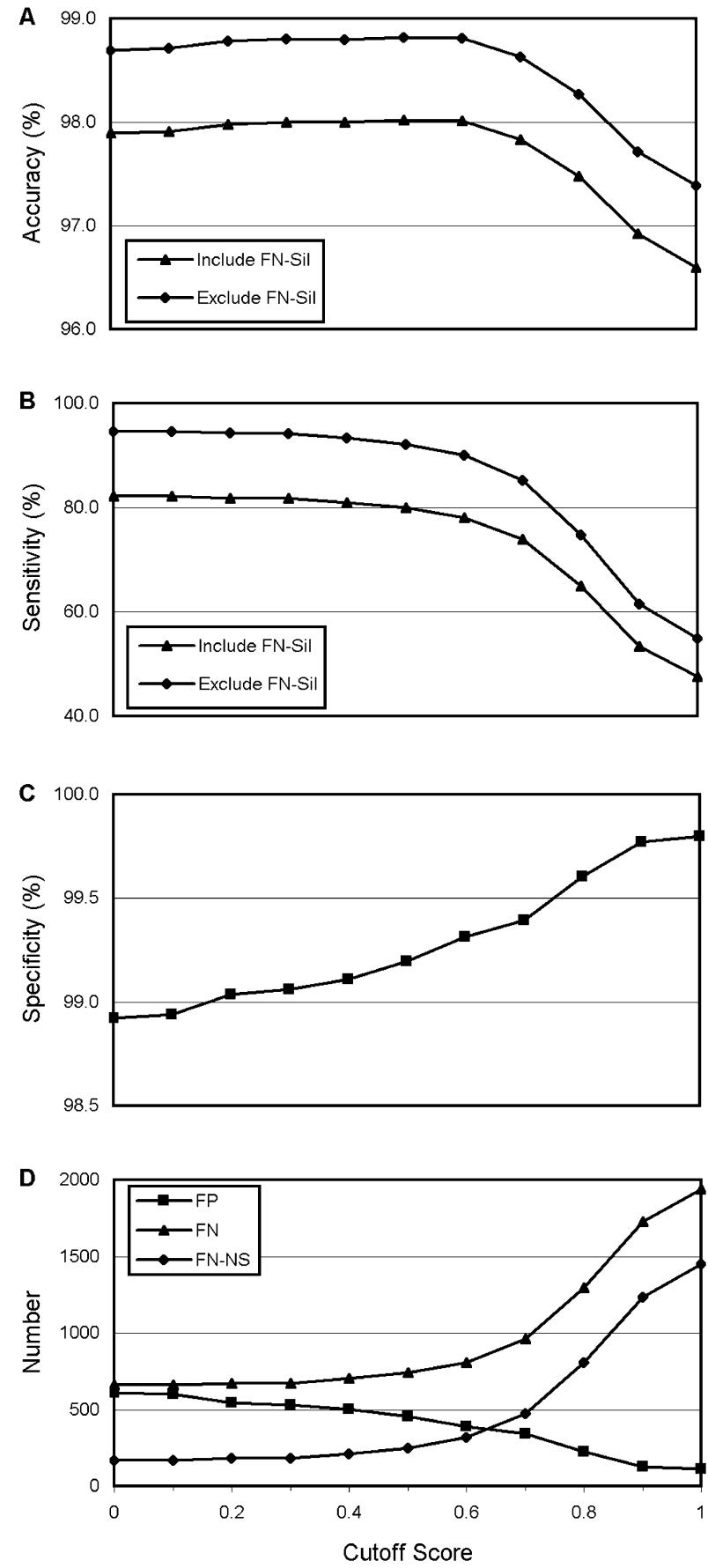

Figure 2

Effect of the cutoff score on PREP-Mt predictive performance. Effect of the cutoff score as measured by $(A)$ accuracy, (B) sensitivity, (C) specificity, and (D) the numbers of false positives (square), false negatives (triangle), and nonsilent false negatives which change the encoded amino acid (diamond). Accuracy and sensitivity were calculated using either all false negatives (triangle) or only the false negatives that change the encoded amino acid (diamond). Cutoff values used for testing ranged from 0 to 1.0 at all increments of 0.1 . proteins. Because of this, numerous sites of RNA editing were incorrectly inferred in these regions. Interestingly, the actual number of true editing sites in these same regions is extremely low, with less than one site observed on average in extensions that can span up to 1,000 nucleotides in length. Therefore, these non-homologous extensions can be removed from the ASD with almost no negative consequences.

Accuracy is also consistently high, which is due to the strong influence of specificity on the accuracy score. Accuracy is a measure of overall performance, combining the individual performances of predicting the known unedited sites (measured by specificity) and the known edited sites (measured by sensitivity). However, since the number of known unedited sites vastly outnumbers the number of known edited sites regardless of gene or genus examined, PREP-Mt's ability to identify the known unedited sites is more heavily weighted in the accuracy score. To get an unbiased measure of overall performance, sensitivity and specificity can be averaged. This balanced accuracy value represents the (biologically unrealistic) scenario in which the numbers of known unedited and edited sites are equal. Since specificity is relatively constant across all treatments, the balanced accuracy statistic correlates most strongly with the fluctuations in sensitivity.

PREP-Mt is also very sensitive in identifying the known editing sites that change the encoded amino acid, finding $95 \%$ of these non-silent editing sites. Some of the missed non-silent editing sites are likely lineage-specific gains of RNA editing that do not get predicted because the lineage is underrepresented in the ASD. Others are probably due to errors in the data that defines the known edited and unedited sites (see below). Analysis of sensitivity reveals that the main limitation of the PREP-Mt methodology is its inability to identify silent editing sites. Currently, the tie-breaking rules used by PREP-Mt always select the codon state that requires the fewest number of edits. Since any codon with the potential for silent editing produces the same amino acid regardless of editing status, the scores for the edited and unedited states will be identical and the tie-breaking rule will always select the unedited state. It would be possible to change this tie-breaking rule so that the state with the most number of edits is preferentially chosen, but doing so would lead to an overwhelming number of false positives at these potential silent editing sites, since the vast majority of these sites are not actually edited. Aligning DNA sequences instead of proteins would help to identify a number of these silent editing sites, but the chances of identifying false positives would be much greater here as well since there are only four different nucleotides in DNA as opposed to 20 amino acids in proteins. Determination of sequence motifs that 
unambiguously specify a particular cytidine as a site of editing would also help to predict these problematic sites. However, even if such motifs exist and are discovered, silent editing sites may remain difficult to predict. Silent editing sites are often found only occasionally edited in experimental studies, suggesting that the putative motifs are more weakly conserved for many silent editing sites.

Unlike the problem of silent editing site prediction, which is due to a real limitation of the methodology, PREP-Mt predictive performance is also negatively affected by errors in the data used to define the known edited and unedited sites, which causes some correctly predicted sites to be incorrectly classified as false positives or false negatives. In experimental determination of RNA editing sites, several types of errors are produced. In some cases, edited sites are not detected, either because a particular site is only occasionally edited or because incompletely edited transcripts are preferentially amplified during PCR. Thus, some predicted editing sites may in fact be true editing sites, but since they were not detected in the experimental analysis they are identified as false positives. In other cases, errors introduced during reverse transcription or sequencing lead to identification of $\mathrm{C}$ to $\mathrm{U}$ changes that are not actual editing sites. Inclusion of these errors in the known set of editing sites causes predicted unedited sites to be incorrectly identified as false negatives. Other noise in the known editing data comes from the fact that the Genbank annotations are subject to human error. Numerous examples of annotation mistakes were encountered while constructing the Aligned Sequence Database. Most were easily corrected because they involved a nucleotide that was not a cytidine. While three cases were known sites of the rare $\mathrm{U}$ to $\mathrm{C}$ editing in angiosperms, the vast majority were clearly mistakes. It is expected that about $25 \%$ of annotation errors would by chance happen to annotate another cytidine, and these are undetectable except by cross-referencing every known edited site with the literature. Since this was not done, a small increase in both false positives and false negatives is expected. Including sequences from Marchantia, Chara, and Chaetosphaeridium, which may actually perform RNA editing to some extent, and from Table 3, which may simply be the result of incomplete experimental analysis, could also cause predicted editing sites to be incorrectly classified as false positives.

\section{Improving predictive performance}

Because the current prediction scheme calculates the score using all ASD homologues, lineage-specific gains or losses of editing may be missed due to the skewed phylogenetic distribution of sequences in the ASD (Fig. 3). Although mitochondrial RNA editing is known to occur in almost all major land plant lineages [4-6], 74\% of the proteins in the ASD were from the angiosperms, while almost all of the other proteins came from organisms that most likely

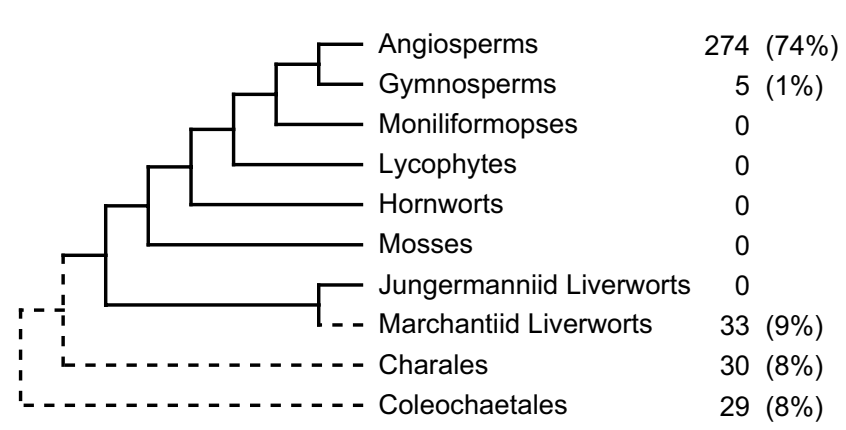

Figure 3

Skewed phylogenetic distribution of sequences in the Aligned Sequence Database. Shown are the number of sequences in the ASD for each lineage and the percentage of the total number of sequences. Editing information is available for several genes from the underrepresented groups, but they were not included in the ASD because only portions of the genes were analyzed. The dotted lines indicate lack of RNA editing based on previous predictive and experimental analyses (see main text). The marchantiid liverworts are thought to have secondarily lost the ability to perform RNA editing. The relationship between plant lineages was taken from Knoop [33].

do not perform RNA editing. In contrast, only five sequences were available from the gymnosperms, and the other major plant lineages were not represented at all. Partial sequence data with editing information was available for a number of genes from the underrepresented groups; however, these sequences covered less than half of the gene in almost all cases and were therefore not included in the ASD.

One way to overcome the skewed sample of ASD sequences would be to modify the scoring scheme so that it approximates phylogenetic methods. Because RNA editing sites are heritable, more sites are shared between closely related species than between distantly related ones. Thus, instead of using all of the homologous ASD sequences, a subset of sequences that are closely related to the input sequence could be specified. Assigning sequence weights to the database homologues based on their overall similarity to the input sequence would achieve comparable results. The best solution to this problem would be to use actual phylogenetic methods to identify the most parsimonious or most likely state for a particular codon in the input sequence; however, the speed of prediction would certainly suffer if this strategy was implemented. An alternative approach to overcome the skewed distribution of ASD sequences would be to expand the database so that it consists of a more balanced diversity of species. This approach would also help to reduce the negative 
effects of small sample sizes on predictive performance. As additional mitochondrial genome and transcriptome data become available, it is expected that PREP-Mt performance will continue to increase.

The simplistic scoring scheme may also lead to spurious predictions in poorly conserved protein regions. To address this issue, a component that measures the conservation in the database sequences for each column in the alignment could be incorporated into the score. Regions of high conservation between homologues are often functionally important for that protein. Thus, the correcting nature of RNA editing is expected to be most valuable in these regions and the PREP-Mt prediction scores could be weighted based on the level of alignment conservation.

\section{Comparison to other prediction methods}

A recent paper used sequence context and the estimated folding energy of these regions to predict editing sites in plant mitochondrial genes [24]. The authors constructed a data set containing all of the known edited sites from the complete genomes of Arabidopsis thaliana, Brassica napus, and Oryza sativa and compared them to an equal number of known unedited sites randomly selected from these genomes. Using tree-based statistical models, the combined data set was partitioned based on the variables that showed the greatest ability to discriminate between edited and unedited sites. Editing sites were predicted with $70.5 \%$ accuracy using a simple single-tree approach and $84.8 \%$ accuracy using a "random forest" method that analyzed thousands of trees. These values are directly comparable to the balanced accuracy of $90.5 \%$ for PREP-Mt, since the balanced accuracy statistic is the accuracy expected for PREP-Mt if an equal number of known edited and unedited sites were used. The speed of the tree-based statistical models were not presented, but they are very unlikely (especially the random forest model) to be competitive with the nearly instantaneous predictive results from PREP-Mt.

It should be noted that Cummings and Myers [24] also calculated the sensitivity and specificity of their approach; however, they classified false positives and false negatives differently than what is reported for PREP-Mt. Cummings and Myers classified false negatives as the unedited sites that were incorrectly partitioned with the true positives (the correctly classified edited sites) and false positives as the edited sites that were incorrectly partitioned with the true negatives (the correctly classified unedited sites). This has no effect on the calculation of accuracy, but it has a major effect on sensitivity and specificity scores. For their single-tree approach, Cummings and Myers provided the raw numbers of correctly and incorrectly classified edited and unedited positions, making it possible to calculate specificity and sensitivity in the same way as for PREP-Mt.
This method correctly classified 1,262 out of 1,347 known editing sites (sensitivity $=93.7 \%$ ), but only 637 out of 1,347 known unedited sites (specificity $=47.3 \%$ ). Thus, on the one hand, their approach is very good at identifying RNA edited sites. However, approximately half of all known unedited sites are also incorrectly classified as edited sites. Because their model uses the unrealistic scenario in which the numbers of edited sites and unedited sites are equal, this problem would be increased significantly when applied to biologically realistic situations. For the 1,347 known edited sites that the authors examined from Arabidopsis, Brassica, and Oryza, there are actually about 17,900 cytidines that are not edited $(T N+F P$ from Table 2). Using their single-tree approach, over 9,000 of these unedited cytidines would be incorrectly classified as editing sites. For the random forest model, actual numbers of correctly and incorrectly classified sites were not provided, so similar calculations cannot be determined. However, because their value that incorporates the number of incorrectly partitioned unedited sites is lowest for both approaches, it seems likely that the random forest approach will also identify a substantial number of false positives in biologically realistic situations.

A second recent paper used homologous protein alignments (as does PREP-Mt) to predict C insertion editing sites in the slime mold Physarum polycephalum [37]. Bundschuh predicted RNA editing sites for six Physarum mitochondrial genes by determining their optimal states from a modified hidden markov model (HMM) that allows for cytidine insertions. Transition parameters in the HMM were defined for each gene using the position-specific scoring matrix (PSSM) from a PSI-BLAST alignment of all homologous proteins in Genbank's non-redundant database. Compared to PREP-Mt, the HMM approach was less sensitive in finding editing sites $(71 \%$ on average vs. $82 \%$ for PREP-Mt) and less accurate in determining the correct amino acid sequences ( $92 \%$ on average vs. $99 \%$ for PREPMt [data not shown]). These comparisons of performance should be taken with caution since the two methods were used to predict different types of RNA editing in different organismal lineages. To allow for a more direct comparison, both approaches could be easily modified to predict the other type of editing. However, Bundschuh's use of all homologous proteins to define the PSSM may be problematic if applied to $\mathrm{C}$ to $\mathrm{U}$ editing in plant mitochondria. A significant percentage of homologues identified for a plant mitochondrial protein would be other plant mitochondrial proteins, but almost all of these sequences in Genbank are predictions based on genomic DNA. In other words, RNA editing information is not incorporated or not known and so the predicted protein sequences are not correct. Prediction of editing will get misled by inclusion of these incorrect proteins since many 
editing sites are shared between plants. Additionally, inclusion of non-plant homologues to define the PSSM will lead to more variable alignments, increasing the chances of spurious prediction. It will also make the beginning and end of genes more difficult to align, as already observed for Bundschuh's method which could not analyze approximately $10 \%$ of each Physarum gene. The PREP-Mt method gets around these problems by limiting the sequences to plants, so that the alignments are highly conserved and the protein extremities are easily aligned (except for atp6 and $r p s 2$ which have non-homologous $5^{\prime}$ and $3^{\prime}$ extensions for many species). Furthermore, only correct protein sequences are used in the alignments, since PREP-Mt limits the plant sequences to those with known editing information. Finally, because these alignments are predefined for PREP-Mt, Bundschuh's approach would need to use predefined HMMs for each gene to be competitive in terms of speed.

\section{Applications}

The main use of PREP-Mt will be to identify RNA editing sites in the thousands of plant mitochondrial gene sequences available in the sequence databases, since editing information is only known for a small percentage of these sequences. Furthermore, as many additional plant mitochondrial genome sequencing projects are planned or already underway, PREP-Mt could serve an important role by quickly and accurately determining most sites of RNA editing without the need for sequencing of mitochondrial transcripts.

Currently, RNA editing information for full-length plant mitochondrial genes is limited mostly to the angiosperms (Fig. 3), which almost always convert in the $\mathrm{C}$ to $\mathrm{U}$ direction. Because of this, the reverse $\mathrm{U}$ to $\mathrm{C}$ editing phenomenon was not considered here. However, reverse editing has been found to be much more common in fern and hornwort mitochondria and was shown to increase protein conservation as well [4-6]. It is likely, therefore, that modification of PREP-Mt to allow for $U$ to $\mathrm{C}$ prediction would be successful in the species that regularly perform this type of editing. Similarly, the PREP-Mt methodology could be applied to the problem of RNA editing in plant chloroplasts, which also perform editing that leads to an increase in protein conservation across species [7,11-15]. More generally, the PREP-Mt methodology should be effective for any system where RNA editing increases protein conservation across species.

PREP-Mt could also be used as a biological tool beyond simply identifying sites of RNA editing. For example, PREP-Mt could be used as a determinant of gene functionality. Unlike animals and fungi, whose mitochondrial gene content has remained stable for tens to hundreds of millions of years, gene content in plant mitochondrial genomes is much more variable [33]. The lineage-specific differences in plant mitochondrial gene content are mostly due to the proclivity of some genes to be relocated to the nuclear genome [38]. Upon transfer to the nucleus, the mitochondrial gene copy often degrades into a pseudogene. Because these pseudogenes are often still transcribed and edited [8,9,19-21], non-functionality is ascribed based on the presence of internal stop codons or frameshifts. This could be problematic since a mitochondrial gene, which has been functionally replaced by a nuclear gene, may still be intact and in-frame. Using PREP-Mt, pseudogenes could also be identified based on the fact that their editing positions do not always lead to the increased protein sequence conservation across species that functional genes demonstrate [19-21]. Intact and in-frame genes that demonstrate unusual editing properties, such as those listed in Table 3, may indicate loss of functionality and the presence of a functional nuclear copy, as already suggested for rps1 from Oenothera [39] and $r p s 14$ from Brassica [10]. It is interesting to note that 10 of the 12 cases in Table 3 are from genes that are very often found transferred to the nucleus in plants [38].

For atp1 and cox3, pseudogenization is not a likely hypothesis because these genes have never been found transferred to the nucleus in plants [38]. These two examples (as well as the 10 discussed above) could represent bona fide cases of functional mitochondrial transcripts that do not get edited properly for some reason. Identification of genes with abnormal editing patterns and further analysis into the causes underlying these patterns could lead to an understanding of the mechanism of RNA editing in plants, which is still largely unknown $[2,3]$.

\section{Conclusion}

PREP-Mt is available as an online resource that predicts sites of $\mathrm{C}$ to U RNA editing in plant mitochondrial protein-coding genes. It was tested on a comprehensive set of genes with known RNA editing information and was shown to be highly sensitive, specific, and accurate in most cases. The speed of prediction was also extremely fast. Thus, PREP-Mt is a substantial improvement over other RNA editing prediction methods, and its predictive performance is expected to continue to improve as more editing data become available. PREP-Mt may be useful for predicting protein sequences, for determining gene functionality, and for understanding the mechanism of RNA editing. The PREP-Mt methodology could be used to predict editing sites in any system where the effect of editing is to increase protein conservation across species, such as for reverse $\mathrm{U}$ to $\mathrm{C}$ editing in plants and for plastid RNA editing. 
Availability and requirements

PREP-Mt is an online tool that is freely available for use at http://www.prep-mt.net/

\section{Additional material}

\section{Additional File 1}

atp1 alignment in FASTA format

Click here for file

[http://www.biomedcentral.com/content/supplementary/1471-

2105-6-96-S1.fas]

\section{Additional File 2}

atp4 alignment in FASTA format

Click here for file

[http://www.biomedcentral.com/content/supplementary/1471-

2105-6-96-S2.fas]

\section{Additional File 3}

atp6 alignment in FASTA format

Click here for file

[http://www.biomedcentral.com/content/supplementary/14712105-6-96-S3.fas]

\section{Additional File 4}

atp8 alignment in FASTA format

Click here for file

[http://www.biomedcentral.com/content/supplementary/14712105-6-96-S4.fas]

\section{Additional File 5}

atp9 alignment in FASTA format

Click here for file

[http://www.biomedcentral.com/content/supplementary/14712105-6-96-S5.fas]

\section{Additional File 6}

ccmB alignment in FASTA format

Click here for file

[http://www.biomedcentral.com/content/supplementary/14712105-6-96-S6.fas]

\section{Additional File 7}

\section{ccmC alignment in FASTA format}

Click here for file

[http://www.biomedcentral.com/content/supplementary/14712105-6-96-S7.fas]

\section{Additional File 8}

\section{ccmFc alignment in FASTA format}

Click here for file

[http://www.biomedcentral.com/content/supplementary/1471-

2105-6-96-S8.fas]

\section{Additional File 9}

\section{ccmFn alignment in FASTA format}

Click here for file

[http://www.biomedcentral.com/content/supplementary/14712105-6-96-S9.fas]

\section{Additional File 10}

cob alignment in FASTA format

Click here for file

[http://www.biomedcentral.com/content/supplementary/14712105-6-96-S10.fas]

\section{Additional File 11}

cox1 alignment in FASTA format

Click here for file

[http://www.biomedcentral.com/content/supplementary/14712105-6-96-S11.fas]

\section{Additional File 12}

cox2 alignment in FASTA format

Click here for file

[http://www.biomedcentral.com/content/supplementary/14712105-6-96-S12.fas]

\section{Additional File 13}

\section{cox3 alignment in FASTA format}

Click here for file

[http://www.biomedcentral.com/content/supplementary/1471-

2105-6-96-S13.fas]

\section{Additional File 14}

matR alignment in FASTA format

Click here for file

[http://www.biomedcentral.com/content/supplementary/1471-

2105-6-96-S14.fas]

\section{Additional File 15}

$\mathrm{mttB}$ alignment in FASTA format

Click here for file

[http://www.biomedcentral.com/content/supplementary/1471-

2105-6-96-S15.fas]

\section{Additional File 16}

nad1 alignment in FASTA format

Click here for file

[http://www.biomedcentral.com/content/supplementary/1471-

2105-6-96-S16.fas]

\section{Additional File 17}

nad2 alignment in FASTA format

Click here for file

[http://www.biomedcentral.com/content/supplementary/14712105-6-96-S17.fas]

\section{Additional File 18}

nad3 alignment in FASTA format

Click here for file

[http://www.biomedcentral.com/content/supplementary/14712105-6-96-S18.fas]

\section{Additional File 19}

nad4 alignment in FASTA format

Click here for file

[http://www.biomedcentral.com/content/supplementary/14712105-6-96-S19.fas] 


\section{Additional File 20}

nad4L alignment in FASTA format

Click here for file

[http://www.biomedcentral.com/content/supplementary/14712105-6-96-S20.fas]

\section{Additional File 21}

nad5 alignment in FASTA format

Click here for file

[http://www.biomedcentral.com/content/supplementary/14712105-6-96-S21.fas]

\section{Additional File 22}

nad6 alignment in FASTA format

Click here for file

[http://www.biomedcentral.com/content/supplementary/14712105-6-96-S22.fas]

\section{Additional File 23}

nad7 alignment in FASTA format

Click here for file

[http://www.biomedcentral.com/content/supplementary/14712105-6-96-S23.fas]

\section{Additional File 24}

nad9 alignment in FASTA format

Click here for file

[http://www.biomedcentral.com/content/supplementary/14712105-6-96-S24.fas]

\section{Additional File 25}

rpl2 alignment in FASTA format

Click here for file

[http://www.biomedcentral.com/content/supplementary/14712105-6-96-S25.fas]

\section{Additional File 26}

rpl5 alignment in FASTA format

Click here for file

[http://www.biomedcentral.com/content/supplementary/14712105-6-96-S26.fas]

\section{Additional File 27}

rpl6 alignment in FASTA format

Click here for file

[http://www.biomedcentral.com/content/supplementary/14712105-6-96-S27.fas]

\section{Additional File 28}

rpl16 alignment in FASTA format

Click here for file

[http://www.biomedcentral.com/content/supplementary/14712105-6-96-S28.fas]

\section{Additional File 29}

rps1 alignment in FASTA format

Click here for file

[http://www.biomedcentral.com/content/supplementary/1471-

2105-6-96-S29.fas]

\section{Additional File 30}

rps2 alignment in FASTA format

Click here for file

[http://www.biomedcentral.com/content/supplementary/14712105-6-96-S30.fas]

\section{Additional File 31}

rps3 alignment in FASTA format

Click here for file

[http://www.biomedcentral.com/content/supplementary/14712105-6-96-S31.fas]

\section{Additional File 32}

rps4 alignment in FASTA format

Click here for file

[http://www.biomedcentral.com/content/supplementary/14712105-6-96-S32.fas]

\section{Additional File 33}

rps7 alignment in FASTA format

Click here for file

[http://www.biomedcentral.com/content/supplementary/1471-

2105-6-96-S33.fas]

\section{Additional File 34}

rps8 alignment in FASTA format

Click here for file

[http://www.biomedcentral.com/content/supplementary/1471-

2105-6-96-S34.fas]

\section{Additional File 35}

rps10 alignment in FASTA format

Click here for file

[http://www.biomedcentral.com/content/supplementary/1471-

2105-6-96-S35.fas]

\section{Additional File 36}

rps11 alignment in FASTA format

Click here for file

[http://www.biomedcentral.com/content/supplementary/1471-

2105-6-96-S36.fas]

\section{Additional File 37}

rps12 alignment in FASTA format

Click here for file

[http://www.biomedcentral.com/content/supplementary/14712105-6-96-S37.fas]

\section{Additional File 38}

rps13 alignment in FASTA format

Click here for file

[http://www.biomedcentral.com/content/supplementary/14712105-6-96-S38.fas]

\section{Additional File 39}

rps14 alignment in FASTA format

Click here for file

[http://www.biomedcentral.com/content/supplementary/14712105-6-96-S39.fas] 


Additional File 40
rps19 alignment in FASTA format
Click here for file
[http://www.biomedcentral.com/content/supplementary/1471-
2105-6-96-S40.fas]
Additional File 41
sdh3 alignment in FASTA format
Click here for file
[http://www.biomedcentral.com/content/supplementary/1471-
2105-6-96-S41.fas]
Additional File 42
sdh4 alignment in FASTA format
Click here for file
[http://www.biomedcentral.com/content/supplementary/1471-
2105-6-96-S42.fas]

\section{Acknowledgements}

I thank Jeffrey Palmer, Danny Rice, Aaron Richardson, and Predrag Radivojac for helpful discussions and Alejandro Araya for providing the RNA editing positions in exon 5 of nad $l$ for Triticum aestivum. This research was supported by National Institutes of Health Grant ROI-GM-35087 (to Jeffrey D. Palmer).

\section{References}

I. Benne R, Van den Burg J, Brakenhoff JP, Sloof P, Van Boom JH, Tromp MC: Major transcript of the frameshifted coxll gene from trypanosome mitochondria contains four nucleotides that are not encoded in the DNA. Cell 1986, 46:819-826.

2. Brennicke A, Marchfelder A, Binder S: RNA editing. FEMS Microbiol Rev 1999, 23:297-316.

3. Mulligan RM: RNA editing in plant organelles. In Molecular Biology and Biotechnology of Plant Organelles Edited by: Daniel H, Chase CD. Dordrecht: Kluwer; 2004:239-260.

4. Hiesel R, Combettes B, Brennicke A: Evidence for RNA editing in mitochondria of all major groups of land plants except the Bryophyta. Proc Natl Acad Sci USA 1994, 91:629-633.

5. Malek O, Lättig K, Hiesel R, Brennicke A, Knoop V: RNA editing in bryophytes and a molecular phylogeny of land plants. EMBO J 1996, I5:|403-1411.

6. Steinhauser S, Beckert S, Capesius I, Malek O, Knoop V: Plant Mitochondrial RNA Editing. J Mol Evol 1999, 48:303-312.

7. Freyer R, Kiefer-Meyer MC, Kössel H: Occurrence of plastid RNA editing in all major lineages of land plants. Proc Natl Acad Sci USA 1997, 94:6285-6290.

8. Giegé $P$, Brennicke A: RNA editing in Arabidopsis mitochondria effects 44 I C to U changes in ORFs. Proc Natl Acad Sci USA 1999, 96: $15324-15329$.

9. Notsu Y, Masood S, Nishikawa T, Kubo N, Akiduki G, Nakazono M, Hirai A, Kadowaki K: The complete sequence of the rice (Oryza sativa. L.) mitochondrial genome: frequent DNA sequence acquisition and loss during the evolution of flowering plants. Mol Genet Genomics 2002, 268:434-445.

10. Handa $\mathrm{H}$ : The complete nucleotide sequence and RNA editing content of the mitochondrial genome of rapeseed (Brassica napus L.): comparative analysis of the mitochondrial genomes of rapeseed and Arabidopsis thaliana. Nucleic Acids Res 2003, 3 1:5907-5916.

I I. Maier RM, Neckermann K, Igloi GL, Kössel H: Complete sequence of the maize chloroplast genome: gene content, hotspots of divergence and fine tuning of genetic information by transcript editing. J Mol Biol 1995, 25 I:6I4-628.

12. Hirose T, Kusumegi T, Tsudzuki T, Sugiura M: RNA editing sites in tobacco chloroplast transcripts: editing as a possible regula- tor of chloroplast RNA polymerase activity. Mol Gen Genet 1999, 262:462-467.

13. Schmitz-Linneweber C, Regel R, Du TG, Hupfer H, Herrmann RG, Maier RM: The plastid chromosome of Atropa belladonna and its comparison with that of Nicotiana tabacum : the role of RNA editing in generating divergence in the process of plant speciation. Mol Biol Evol 2002, I9:1602-1612.

14. Kugita M, Yamamoto Y, Fujikawa T, Matsumoto T, Yoshinaga K: RNA editing in hornwort chloroplasts makes more than half the genes functional. Nucleic Acids Res 2003, 3 I:24I 7-2423.

15. Wolf PG, Rowe CA, Hasebe M: High levels of RNA editing in a vascular plant chloroplast genome: analysis of transcripts from the fern Adiantum capillus-veneris. Gene 2004, 339:89-97.

16. Covello PS, Gray MW: RNA editing in plant mitochondria. Nature 1989, 34 I:662-666.

I7. Gualberto JM, Lamattina L, Bonnard G, Weil JH, Grienenberger JM: RNA editing in wheat mitochondria results in the conservation of protein sequences. Nature 1989, 341:660-662.

18. Hiesel R, Wissinger B, Schuster W, Brennicke A: RNA editing in plant mitochondria. Science I989, 246: I632-I634.

19. Schuster W, Brennicke A: RNA editing makes mistakes in plant mitochondria: editing loses sense in transcripts of a rps 19 pseudogene and in creating stop codons in coxl and rps3 mRNAs of Oenothera. Nucleic Acids Res 1991, 19:6923-6928.

20. Quiñones V, Zanlungo S, Moenne A, Gómez I, Holuigue L, Litvak S, Jordana $X$ : The rpl5-rps/4-cob gene arrangement in Solanum tuberosum: rps 14 is a transcribed and unedited pseudogene. Plant Mol Biol 1996, 3 I:937-943.

21. Grohmann L, Brennicke A, Schuster W: The mitochondrial gene encoding ribosomal protein $S I 2$ has been translocated to the nuclear genome in Oenothera. Nucleic Acids Res 1992, 20:564l-5646.

22. Choury D, Farré JC, Jordana $X$, Araya A: Different patterns in the recognition of editing sites in plant mitochondria. Nucleic Acids Res 2004, 32:6397-6406.

23. Takenaka M, Neuwirt J, Brennicke A: Complex cis-elements determine an RNA editing site in pea mitochondria. Nucleic Acids Res 2004, 32:4137-4I44.

24. Cummings MP, Myers DS: Simple statistical models predict Cto-U edited sites in plant mitochondrial RNA. BMC Bioinformatics 2004, 5: I 32.

25. Gray MW, Covello PS: RNA editing in plant mitochondria and chloroplasts. FASEB J 1993, 7:64-7I.

26. Glaubitz JC, Carlson JE: RNA editing in the mitochondria of a conifer. Curr Genet 1992, 22:163-165.

27. Oda K, Yamato K, Ohta E, Nakamura Y, Takemura M, Nozato N, Akashi K, Kanegae T, Ogura Y, Kohchi T, Ohyama K: Gene organization deduced from the complete sequence of liverwort Marchantia polymorpha mitochondrial DNA: A primitive form of plant mitochondrial genome. J Mol Biol 1992, 223:1-7.

28. Turmel M, Otis C, Lemieux C: The chloroplast and mitochondrial genome sequences of the charophyte Chaetosphaeridium globosum : insights into the timing of the events that restructured organelle DNAs within the green algal lineage that led to land plants. Proc Natl Acad Sci USA 2002, 99: I I275-I I 280.

29. Turmel M, Otis C, Lemieux C: The mitochondrial genome of Chara vulgaris : insights into the mitochondrial DNA architecture of the last common ancestor of green algae and land plants. Plant Cell 2003, I 5: I888-1903.

30. Ohyama K, Oda K, Ohta E, Takemura M: Gene organization and evolution of introns of a liverwort, Marchantia polymorpha, mitochondrial genome. In Plant Mitochondria Edited by: Brennicke A, Kück U. Weinheim: Verlag Chemie; 1993:II5-129.

31. predictive RNA editor for plant mitochondrial genes [http:// www.prep-mt.net/]

32. Thompson JD, Higgins DG, Gibson TJ: CLUSTAL W: improving the sensitivity of progressive multiple sequence alignment through sequence weighting, positions-specific gap penalties and weight matrix choice. Nucleic Acids Res 1994, 22:4673-4680.

33. Knoop V: The mitochondrial DNA of land plants: peculiarities in phylogenetic perspective. Curr Genet 2004, 46: I23-139.

34. Wolfe KH, Li WH, Sharp PM: Rates of nucleotide substitution vary greatly among plant mitochondrial, chloroplast, and nuclear DNAs. Proc Natl Acad Sci USA 1987, 84:9054-9058. 
35. Palmer JD, Herbon LA: Plant mitochondrial DNA evolves rapidly in structure, but slowly in sequence. J Mol Evol 1988, 28:87-97.

36. Cho Y, Mower JP, Qiu YL, Palmer JD: Mitochondrial substitution rates are extraordinarily elevated and variable in a genus of flowering plants. Proc Natl Acad Sci USA 2004, I 0 I: I 774 |- 17746.

37. Bundschuh R: Computational prediction of RNA editing sites. Bioinformatics 2004, 20:3214-3220.

38. Adams KL, Qiu YL, Stoutemyer M, Palmer JD: Punctuated evolution of mitochondrial gene content: high and variable rates of mitochondrial gene loss and transfer to the nucleus during angiosperm evolution. Proc Natl Acad Sci USA 2002, 99:9905-991 2.

39. Mundel C, Schuster W: Loss of RNA editing of rps I sequences in Oenothera mitochondria. Curr Genet 1996, 30:455-460.

Publish with Bio Med Central and every scientist can read your work free of charge

"BioMed Central will be the most significant development for disseminating the results of biomedical research in our lifetime. "

Sir Paul Nurse, Cancer Research UK

Your research papers will be:

- available free of charge to the entire biomedical community

- peer reviewed and published immediately upon acceptance

- cited in PubMed and archived on PubMed Central

- yours - you keep the copyright

Submit your manuscript here:

http://www.biomedcentral.com/info/publishing_adv.asp
BioMedcentral 